\title{
Mortality and risk factors for late death in tetralogy of Fallot: the Japanese Nationwide Multicenter Survey
}

$\mathrm{N}$ IWA AND Associates ${ }^{1}$ Have published a remarkable paper on late results of tetralogy of Fallot, the only relevant issue beyond operative survival. Obviously the division in early versus late survival constitutes an artificial dichotomy, and limitation to one of the two results represents turning a blind eye to the other. Most medical papers are limited to early results only, and are therefore limited in their long term inferences. In contrast, only a few papers are limited exclusively to late results, which blinds us for the early period, defined as hospital survival in the study of Niwa and colleagues. ${ }^{1}$ Hospital survival in itself is not limited by time, and thus is limited in its applicability. The question then arises to what extent this blinded period influences our interpretation of the late results? The paucity of data related to details of patients and their treatment in this paper is unfortunate. It is understandable, nonetheless, that historic data up to 30 years old from 12 participating centres is extremely difficult, possibly impossible, to validate. The current paper is restricted to late results, and therefore our inferences as to the factors involving patients and operative risk is limited, whilst their degree of completeness is not entirely clear.

The net result is that the three-tenths of the cohort initially undergoing surgery in 1972 had died within one year, with an additional fifth either lost to follow-up, or excluded because of incomplete data. The population studied, therefore, is only half of the initial number. For the 1982 cohort, the 1-year mortality is $14 \%$, and $13 \%$ is lost to follow-up or excluded, giving a population studied of almost threequarters. The decrease in mortality is to be applauded, but the substantial number of patients that still disappeared from the study is worrying.

Of the purported "top 20 institutions" in Japan, 12 joined the current study. This, of course, immediately poses the question of the criterions used for choice of a "top institution", and why the other 8 centres chose not to join. Bias in favour of the participants is an obvious possibility. Additionally, it is not clear why "bospital" (or "surgeon") was not included in the logistic regression analysis, but was instead analysed separately. In many other studies, the hospital centre itself almost invariably proved to be a risk factor. When presented in anonymised fashion, hospitals should have no qualms about their results being compared with the others.

What, then, are the conclusions that can be drawn from the present study? Obviously early mortality has halved in the interval of 10 years, which is wonderful, and in keeping with international developments. In the multivariate analysis, older age, and reoperation, were risk factors for late death. The age at operation of both cohorts is so high, however, that the range is not quite representative of the current era. The most striking finding is that there was no reoperation for pulmonary regurgitation at all, in contrast to the experience in many other centres. This is the more surprising when account is taken of the widely used practice of transannular patching in the Japanese series. The role played by the monocusp pulmonary valve, and its extent, cannot be deduced from this paper. It is not clear in what proportion of patients this valve was used, and in what proportion it still functions. My personal experience is that the valve often ceases functioning within a year after operation.

Our Japanese colleagues, nonetheless, are to be complimented for this straightforward and honest report of data. It is as close to a cross-sectional epidemiological approach as is apparently possible. The resulting impression is encouraging. Improvements over time are striking, and the insights provided offer an interesting feedback upon our current practice.

$$
\begin{array}{r}
\text { Tjark Ebels } \\
\text { Chief, Thoraxcentrum } \\
\text { Academisch Ziekenhuis Groningen, Groningen } \\
\text { The Netherlands } \\
\text { E-mail: T.Ebels@thorax.azg.nl }
\end{array}
$$

\section{Reference}

1. Niwa K, Hamada H, Nakazawa M, et al, for the Japanese Multicenter Study Group on Long-term Follow-up of Postoperative Tetralogy of Fallot. Mortality and risk factors for late deaths in tetralogy of Fallot - the Japanese nationwide multicenter survey. Cardiol Young 2002; 12: 453-460. 